
las Ciencias

ISSN: 1697-011X

revista.eureka@uca.es

Universidad de Cádiz

España

\title{
Diseño de una secuencia topocéntrica de enseñanza-aprendizaje de las estaciones del año para nivel primario
}

\section{Galperin, Diego}

Diseño de una secuencia topocéntrica de enseñanza-aprendizaje de las estaciones del año para nivel primario Revista Eureka sobre Enseñanza y Divulgación de las Ciencias, vol. 19, núm. 1, 2022 Universidad de Cádiz, España

Disponible en: https://www.redalyc.org/articulo.oa?id=92068491005

DOI: https://doi.org/10.25267/Rev_Eureka_ensen_divulg_cienc.2022.v19.i1.1801 


\section{Diseño de una secuencia topocéntrica de enseñanza-aprendizaje de las estaciones del año para nivel primario}

Design of a topocentric teaching-learning sequence about seasons for primary education

Diego Galperin

Sede Andina, Universidad Nacional de Rio Negro,

Bariloche, Argentina

dgalperin@unrn.edu.ar

iD https://orcid.org/0000-0002-0705-818X
DOI: https://doi.org/10.25267/

Rev_Eureka_ensen_divulg_cienc.2022.v19.11.1801 Redalyc: https://www.redalyc.org/articulo.oa? $\mathrm{id}=92068491005$

Recepción: 21 Marzo 2021

Revisado: 03 Mayo 2021

Aprobación: 28 Julio 2021

\section{Resumen:}

Este estudio describe el proceso de diseño y evaluación de una secuencia de enseñanza-aprendizaje sobre el fenómeno de las estaciones del año a partir de la implementación de una investigación basada en el diseño. La propuesta fue diseñada utilizando el sistema de referencia topocéntrico e implementada como etapa inicial de la investigación en un curso de sexto año de educación primaria. Los resultados obtenidos parecen indicar que la propuesta resulta viable para lograr una comprensión adecuada del fenómeno desde un punto de vista local. Se presentan conclusiones para el rediseño de la secuencia para futuras implementaciones y algunos aportes de esta investigación para la enseñanza de la astronomía en el nivel primario.

Palabras clave: Estaciones del año, Secuencia de enseñanza-aprendizaje, Investigación basada en el diseño, Sistema de referencia topocéntrico, Educación primaria.

\section{Abstract:}

The design and evaluation process of a teaching-learning sequence about the seasons phenomenon using design-based research is described in this study. The proposal was designed using the topocentric frame of reference and implemented as the initial stage of the research in a sixth grade of primary education. The results obtained seem to indicate that the proposal is viable to achieve an adequate understanding of the phenomenon from a local point of view. Conclusions for the redesign of the sequence for future implementations and some contributions of this research for astronomy teaching at primary education are presented.

KEYWORDS: Seasons, Teaching-learning sequence, Design-based research, Topocentric reference frame, Primary education.

\section{INTRODUCCIÓN}

Numerosas investigaciones realizadas en las últimas décadas evidencian que el fenómeno de las estaciones del año es comprendido por una baja proporción de estudiantes de todos los niveles educativos, incluyendo futuros docentes y alumnos universitarios (Baxter 1989, Ojala 1992, Schoon 1992, De Manuel 1995, Sharp 1996, Kikas 1997, Trumper 2001, Danaia y McKinnon 2007, Vílchez-González y Ramos-Tamajón 2015, Alvarez et al. 2018). Estos resultados pueden tener diferentes causas, entre las que se encuentran:

i. Dificultades de comprensión del fenómeno de las estaciones del año por parte de docentes en actividad, similares a las detectadas en los estudiantes (Camino 1995, García Barros et al. 1996, Fernández Nistal y Peña Boone 2007, Galperin et al. 2018).

ii. Presencia de gran cantidad de errores conceptuales y didácticos en los libros de texto, muchos de los cuales refuerzan las concepciones alternativas que poseen los estudiantes sobre la causa de los fenómenos astronómicos (Vega Navarro 1996, Langhi y Nardi 2007, Galperin y Raviolo 2017). Estos mismos errores se encuentran también en vídeos educativos y de divulgación presentes en Internet, los cuales suelen ser utilizados como recursos didácticos por los docentes (Galperin et al. 2020). 
iii. Desconocimiento de investigaciones educativas que evidencian que la comprensión de los fenómenos astronómicos a partir de los movimientos de la Tierra en el espacio con su eje inclinado, y de la Luna en torno a nuestro planeta, requiere conocimientos previos y habilidades visoespaciales, lo que representa una dificultad para estudiantes de todas las edades (Parker y Heywood 1998, García Barros et al. 2003, Black 2005, Gali y Venukapalli 2021).

iv. Utilización preponderante de explicaciones de los fenómenos astronómicos cotidianos desde un sistema de referencia externo a la Tierra: a partir de los movimientos de la Tierra y la Luna vistos desde el espacio exterior, con sus dificultades de comprensión asociadas. Esto deja de lado la posibilidad de brindar explicaciones adecuadas de dichos fenómenos utilizando el sistema de referencia topocéntrico, centrado en un punto de la superficie terrestre (Galperin y Raviolo 2014).

En función de esta problemática se diseñó una secuencia de enseñanza-aprendizaje (SEA) del fenómeno de las estaciones del año para estudiantes de nivel primario con el fin de ser implementada y evaluada en condiciones de aula. La misma posee como eje la vinculación de los estudiantes con los aspectos observables del fenómeno y la utilización del sistema de referencia topocéntrico para explicar dichas observaciones.

Para su diseño, desarrollo y evaluación se ha decidido recurrir al enfoque de Investigación basada en el diseño (IBD), el cual hace uso de diversas herramientas metodológicas con el fin de evaluar las intervenciones educativas en contextos reales y específicos de la propia práctica. De este modo, se centra la mirada en la mejora del conocimiento sobre dichas intervenciones y, al mismo tiempo, sobre su proceso de diseño y desarrollo (Plomp 2010). Con ello se pretende aportar a la mejora de la realidad educativa recurriendo a teorías o modelos científicos disponibles con el fin de resolver problemas concretos en el propio contexto local, sometiéndolos a prueba y dándolos a conocer. Esto implica compartir el diseño didáctico elaborado junto con el marco teórico que lo justifica, brindando descripciones del proceso de implementación y detallando su etapa de evaluación. De este modo, se desarrolla un proceso iterativo de implementaciones en los cuales la teoría se valida en función de su capacidad para explicar y producir cambios en el mundo real (Guisasola, Amettler y Zuza 2020).

Como parte del proceso de diseño se han elaborado y diseñado algunas representaciones visuales que permiten explicar las estaciones del año en forma topocéntrica a partir del movimiento diario y anual del Sol en el cielo. De esta forma, se busca que los estudiantes de nivel primario mejoren su comprensión del fenómeno sin necesidad de tener que alternar entre dos puntos de vista: el externo a la Tierra, con nuestro planeta moviéndose en el espacio con su eje inclinado, y el visible desde su superficie, con el Sol moviéndose en el cielo y cambiando su recorrido diario cada día.

Los aprendizajes logrados a partir de esta propuesta topocéntrica no generan obstáculos epistemológicos o didácticos que podrían dificultar la comprensión del fenómeno de las estaciones del año desde un punto de vista externo a la Tierra. En este sentido, las explicaciones topocéntricas pueden actuar como modelo intermediario (Gilbert y Boulter 2000) hacia la construcción del modelo heliocéntrico.

\section{MARCo TEÓRICo PARA EL DISEÑo DE LA SEA}

En esta sección se detalla la información relevante para comprender el proceso de diseño, implementación y evaluación de la SEA. El mismo comenzó con la realización de una revisión bibliográfica con el fin de conocer las concepciones más comunes de los estudiantes acerca de la causa de las estaciones del año. A partir de los resultados, se analizó la conveniencia de focalizar en explicaciones concretas vinculadas a la observación directa del cielo, por lo que se elaboró un modelo objetivo de la SEA utilizando el sistema de referencia topocéntrico. Posteriormente, se elaboró una secuencia didáctica topocéntrica que fue implementada en un determinado grupo escolar y evaluada con el fin de proponer modificaciones. Este proceso inicial será continuado iterativamente mediante otros sucesivos rediseños e implementaciones. 


\section{Concepciones de estudiantes sobre la causa de las estaciones}

Existen numerosas investigaciones realizadas con estudiantes de distintas edades sobre las explicaciones que dan al fenómeno de las estaciones del año. Las características de algunas de ellas y las conclusiones obtenidas se encuentran detalladas en la tabla 1.

TABLA 1

Concepciones sobre la causa de las estaciones del año detectadas en alumnos de todos los niveles. Síntesis de resultados obtenidos en investigaciones anteriores

\begin{tabular}{|c|c|c|}
\hline Artículo & Muestra & Concepciones detectadas y/o conclusiones \\
\hline $\begin{array}{l}\text { Baxter } \\
(1989)\end{array}$ & $\begin{array}{l}100 \text { estudiantes de } \\
9 \text { a } 16 \text { años } \\
\text { Inglaterra }\end{array}$ & $\begin{array}{l}\text { - } \quad \text { E1 Sol se aleja de la Tierra en invierno (respuesta mayoritaria) } \\
\text { - La inclinación del eje provoca que distintas partes de la Tierra se } \\
\text { encuentren a diferente distancia del Sol }\end{array}$ \\
\hline $\begin{array}{c}\text { Ojala } \\
(1992)\end{array}$ & $\begin{array}{l}87 \text { futuros docentes } \\
\text { Escandinavia }\end{array}$ & $\begin{array}{l}\text { - } \quad \text { La inclinación de los rayos del Sol va cambiando }(51,5 \%) \\
\text { - } \quad \text { Las estaciones ocurren cuando se caen las hojas }(25,3 \%)\end{array}$ \\
\hline $\begin{array}{l}\text { Schoon } \\
(1992)\end{array}$ & $\begin{array}{l}1213 \text { estudiantes de } \\
\text { primaria a superior } \\
\text { Estados Unidos }\end{array}$ & $\begin{array}{l}\text { - La Tierra cambia su distancia al Sol durante el año }(77,6 \%) \\
\text { - } \quad \text { El Sol se pone por el oeste en mayo, junio y julio }(58 \%)\end{array}$ \\
\hline $\begin{array}{l}\text { De Manuel } \\
\text { (1995) }\end{array}$ & $\begin{array}{l}904 \text { estudiantes de } \\
12 \text { a } 18 \text { años } \\
\text { España }\end{array}$ & $\begin{array}{l}\text { - } \quad \text { La Tierra varía su distancia al Sol durante el año }(71,6 \%) \\
\text { - } \quad \text { La Tierra se traslada con su eje inclinado }(10,6 \%) \\
\text { - } \quad \text { Es verano en la parte de la Tierra que apunta al Sol }(7,2 \%)\end{array}$ \\
\hline $\begin{array}{l}\text { Sharp } \\
(1996)\end{array}$ & $\begin{array}{l}42 \text { estudiantes de } \\
10 \text { y } 11 \text { años } \\
\text { Inglaterra }\end{array}$ & $\begin{array}{l}\text { - La Tierra se acerca y se aleja del Sol alternativamente } \\
\text { - La parte de la Tierra que mira hacia el Sol está en verano } \\
\text { - } \quad \text { El Sol varía la cantidad de calor que irradia }\end{array}$ \\
\hline $\begin{array}{l}\text { Kikas } \\
(1997)\end{array}$ & $\begin{array}{l}252 \text { estudiantes de } \\
9 \text { a } 17 \text { años } \\
\text { 3ro., } 5 \text { to., } 7 \text { mo. y } \\
\text { 9no. año } \\
\text { Estonia }\end{array}$ & $\begin{array}{l}\text { - Respuestas descriptivas sobre lo que se ve o se siente sin indicar la } \\
\text { causa ( } 91 \% \text { en 3ro., } 24 \% \text { en } 5 \text { to., } 40 \% \text { en } 7 \text { mo. y } 41 \% \text { en } 9 \text { no) } \\
\text { - Respuesta presente en el libro escolar: traslación de la Tierra con su } \\
\text { eje inclinado ( } 2 \% \text { en 3ro., } 59 \% \text { en } 5 \text { to., } 31 \% \text { en } 7 \text { mo. y } 47 \% \text { en } 9 \text { no) }\end{array}$ \\
\hline $\begin{array}{l}\text { Trumper } \\
(2001)\end{array}$ & $\begin{array}{l}2087 \text { estudiantes de } \\
\text { secundaria a superior } \\
\text { Israel }\end{array}$ & $\begin{array}{l}\text { - La Tierra se acerca al Sol en verano: } 40 \% \text { secundaria, } 37 \% \text { futuros } \\
\text { docentes de primaria, } 32 \% \text { futuros docentes de secundaria y } 32 \% \\
\text { estudiantes de universidad de carreras no científicas }\end{array}$ \\
\hline $\begin{array}{l}\text { Danaia y } \\
\text { McKinnon } \\
\quad(2007)\end{array}$ & $\begin{array}{l}1920 \text { estudiantes de } \\
7 \text { mo. a 9no. grado } \\
\text { Australia }\end{array}$ & $\begin{array}{ll}\text { - } & \text { No responde ( } 55,0 \% \text { en } 7 \text { mo., } 42,8 \% \text { en } 8 \text { vo. y } 44,2 \% \text { en } 9 \text { no.) } \\
\text { - } & \text { Distancia variable Tierra - Sol }(28,1 \%, 33,5 \% \text { y } 28,2 \%) \\
\text { - } & \text { Traslación con el eje inclinado }(1,0 \%, 4,8 \% \text { y } 6,3 \%)\end{array}$ \\
\hline $\begin{array}{l}\text { Vílchez- } \\
\text { González y } \\
\text { Ramos- } \\
\text { Tamajón } \\
\text { (2015) }\end{array}$ & $\begin{array}{l}61 \text { estudiantes de } \\
7 \text { y } 8 \text { años } \\
\text { España }\end{array}$ & $\begin{array}{l}\text { ¿Por qué hace frío en invierno? } \\
-\quad \text { No responde }(57,4 \%) \\
\text { - } \quad \text { Redundantes: "porque es así" }(32,8 \%) \\
\text { - } \quad \text { Vivenciales: "porque si no, nos moriríamos de frío" }(6,5 \%) \\
\text { - } \quad \text { Por la traslación de la Tierra o por la posición del Sol }(3,3 \%)\end{array}$ \\
\hline $\begin{array}{l}\text { Alvarez et } \\
\text { al. }(2018)\end{array}$ & $\begin{array}{l}126 \text { estudiantes de } \\
10 \text { a } 16 \text { años } \\
\text { Argentina }\end{array}$ & $\begin{array}{l}\text { - Las estaciones se deben a cambios en el paisaje: nieve, lluvia, vientos, } \\
\text { caída de hojas, etc ( } 62,2 \% \text { primaria y } 50 \% \text { secundaria) } \\
\text { - } \quad \text { La Tierra cambia su distancia al Sol al trasladarse }(7,3 \% \text { y } 18,2 \%) \\
\text { - Traslación de la Tierra con su eje inclinado }(3,7 \% \text { y } 2,3 \%)\end{array}$ \\
\hline
\end{tabular}


Los resultados anteriores permiten concluir que el fenómeno de las estaciones del año es comprendido por una proporción muy pequeña de estudiantes de todas las edades. Las explicaciones más comunes que aparecen en la mayoría de las investigaciones corresponden a distintos modelos de distancia variable (Navarrete, Azcárete y Oliva 2004), los cuales sostienen que las estaciones se deben a que la distancia al Sol cambia a lo largo del año. A su vez, algunas de las investigaciones muestran que una proporción importante de estudiantes de primaria y secundaria no logra elaborar una explicación del fenómeno, brindando sólo aspectos descriptivos y vivenciales de los cambios a lo largo del año: caída de las hojas, ir a la playa, etc.

\section{Conclusiones del proceso de revisión bibliográfica}

Algunos intentos para explicar las dificultades ya mencionadas han sugerido que las mismas pueden estar relacionadas con la presencia de una única explicación del fenómeno en la mayoría de los libros y recursos audiovisuales: a partir del movimiento de la Tierra en el espacio con su eje siempre igualmente inclinado. Esta explicación requiere habilidades visoespaciales para su comprensión, conocimientos anteriores y la superposición de dos puntos de vista diferentes: el externo a la Tierra junto con el visible desde su superficie. Sin embargo, muchos estudiantes suelen presentar dificultades en la visualización de modelos tridimensionales, y en la representación bidimensional de objetos tridimensionales, por lo que les resulta complejo poder trabajar desde una perspectiva distinta a la correspondiente a un observador terrestre (Parker y Heywood 1998).

En este sentido, no es sencillo relacionar el movimiento de la Tierra con la cantidad de radiación recibida en determinada zona y con su cambio a lo largo del año (Navarrete, Azcárete y Oliva 2004). Esta explicación requiere que el estudiante se posicione imaginariamente fuera de la Tierra y que imagine su desplazamiento alrededor del Sol mientras que, al mismo tiempo, analiza las consecuencias que esto provoca en la altura y en la trayectoria que sigue el Sol en el cielo local. Aunque algunos trabajos proponen la utilización de simulaciones para facilitar la comprensión, éstas pueden generar nuevas representaciones inadecuadas si el alumno aplica literalmente algunos aspectos de la simulación (De Manuel 1995). Por lo tanto, el tratamiento de aspectos avanzados por medio de simulaciones debería ser posterior al aprendizaje de conceptos referentes a la Tierra como cuerpo cósmico, tales como tamaño, forma o inclinación del eje, que muchas veces no son comprendidos ni por estudiantes de los últimos años de nivel primario (Nussbaum 1979). Estos resultados sugieren la necesidad de priorizar los aspectos descriptivos y observacionales en el nivel primario dado que gran parte de los estudiantes suelen finalizar este nivel educativo sin saber dibujar correctamente la trayectoria diaria del Sol en el cielo, o sin haber observado nunca la variación en la elevación del Sol a lo largo del año (Navarro Pastor 2011, Vílchez-González y Ramos-Tamajón 2015).

Dada la complejidad mencionada, se ha cuestionado la enseñanza a niños de nivel primario de los movimientos de los astros en el espacio exterior y, al mismo tiempo, su relación con lo que se observa en el cielo terrestre. Este tipo de explicaciones desde dos puntos de vista requieren habilidades de pensamiento espacial, tales como rotación mental y visualización espacial, las cuales han sido identificadas como factores que permiten explicar las diferencias de comprensión del fenómeno entre los estudiantes (Black 2005, Plummer et al. 2014). Por lo tanto, dado que la construcción de un punto de vista heliocéntrico implica un número complejo de factores, no parece apropiado esperar una comprensión de dicha noción antes de la adolescencia temprana. En contraposición, los estudiantes pueden construir nociones intermedias antes de moverse a un punto de vista heliocéntrico (Baxter 1989, Lanciano 1989). Esto implica la necesidad de desarrollar el tema nuevamente en la escuela media ya que se han detectado graduados universitarios sin una comprensión adecuada del fenómeno de las estaciones (Sneider, Bar y Kavanagh 2011). Estos resultados ponen en cuestionamiento la pertinencia de enseñar en la escuela primaria los fenómenos astronómicos cotidianos desde un punto de vista heliocéntrico. 
En síntesis, es posible afirmar que no existe razón fundamentada para explicar el fenómeno de las estaciones del año a niños de nivel primario exclusivamente desde una perspectiva externa a la Tierra. Los criterios de sencillez, relación con el entorno y la posibilidad concreta de realizar predicciones le brinda ventajas a la utilización de explicaciones basadas en lo que se observa desde la superficie terrestre. Esto no quiere decir abandonar el modelo heliocéntrico, sino colocarlo en el marco que le corresponde: como una posible forma de abordar la explicación de dicho fenómeno, conociendo las dificultades de comprensión que suele traer asociadas (Galperin y Raviolo 2014). En consecuencia, sería deseable una modificación de los materiales curriculares con el fin de ir, paulatinamente, desde un punto de vista topocéntrico en el nivel primario a un punto de vista heliocéntrico en el nivel medio.

\section{Utilización del sistema de referencia topocéntrico}

Pese a lo mencionado anteriormente, la mayoría de los materiales didácticos (libros escolares, diseños curriculares, recursos audiovisuales, etc) presentan los temas de astronomía cotidiana desarrollados en forma casi exclusiva a partir de la descripción de los movimientos de los astros vistos desde el espacio exterior (Galperin et al. 2017 2020). Esto parece sugerir la existencia de un sistema «privilegiado» para describir los fenómenos astronómicos, dejando de lado la posibilidad de utilizar un punto de vista local centrado en determinada posición sobre la superficie terrestre. De este modo, se pierde la oportunidad de desarrollar tópicos como sistemas de referencia, modelos y modelización, de relevancia para la enseñanza de las ciencias y para comprender la forma en que el conocimiento científico se desarrolla, se valida y se va transformando. En consecuencia, el sistema heliocéntrico parecería ser utilizado por razones más culturales que científicas, no muy diferentes de las presentes en la antigüedad cuando el sistema geocéntrico era el vigente (Shen y Confrey 2010). En síntesis, dado que tanto el sistema de referencia heliocén trico como el topocéntrico son capaces de explicar adecuadamente las estaciones del año, el uso de uno u otro dependerá de su simplicidad descriptiva, de su posibilidad de brindar explicaciones precisas a aspectos observables y de su capacidad predictiva en relación con el contexto (Galperin y Raviolo 2015).

Las descripciones y explicaciones topocéntricas no requieren un cambio de punto de vista de los estudiantes dado que se encuentran centradas en la propia posición de un observador. Por lo tanto, el fenómeno de las estaciones del año puede explicarse a partir de describir cómo cambia la posición del Sol en el cielo a medida que pasan los días, provocando cambios continuos en su recorrido diario. Esto no implica en modo alguno la enseñanza del antiguo modelo geocéntrico de universo. Por el contrario, se propone la utilización de conocimientos actualizados sobre la relatividad de los sistemas de referencia, lo que permite la descripción del movimiento del Sol desde un punto de la superficie terrestre, lo que se logra ubicando el origen del sistema de referencia en dicho lugar (Lanciano 1989, Camino 1999, Galperin 2011).

\section{Modelos y modelización en la enseñanza de las ciencias}

La enseñanza y el aprendizaje basados en modelos se encuentran orientados a relacionar las representaciones del mundo que poseen los científicos sobre un determinado fenómeno físico con las que utilizan los docentes en las aulas y, finalmente, con las representaciones del fenómeno que terminan construyendo los estudiantes (Gilbert y Boulter 2000). Al respecto, se han identificado diferencias significativas entre los modelos que utilizan los científicos y los presentes en el alumnado, aunque diversas investigaciones han mostrado la conveniencia de utilizar representaciones propias de la ciencia escolar como paso necesario para aprender la ciencia de los científicos. De este modo, la ciencia escolar no sería una simplificación de la ciencia de los científicos adaptada al nivel de los alumnos, sino que constituye una serie de etiquetas lingüísticas, conceptos y modelos propios que facilitan la comprensión de los fenómenos por parte de los estudiantes. Por lo tanto, el 
concepto de modelo debe ser trabajado en las aulas con el fin de que los estudiantes comprendan que todos los modelos, tanto los utilizados dentro del contexto escolar como los que usan los científicos, son construcciones provisorias y perfectibles en cuya elección intervienen aspectos como la sencillez, el poder explicativo y la riqueza teórica (Galagovsky y Adúriz-Bravo 2001).

La enseñanza basada en modelos se concibe como cualquier implementación consistente en recursos informativos, actividades de aprendizaje y estrategias de enseñanza que procuran facilitar la construcción de modelos mentales (Johnson-Laird 1983), teniendo como meta la mejora en la comprensión de los estudiantes sobre los modelos y su rol en la ciencia. Para ello, se apunta a la construcción de modelos de enseñanza que, en modo similar a como lo hacen los modelos científicos, puedan actuar como mediadores entre la teoría y la realidad (Lombardi 1998). Desde esta perspectiva, el proceso de aprendizaje a largo plazo consiste en recorrer un camino paulatino desde las concepciones que poseen los estudiantes antes de la enseñanza hasta alcanzar el modelo objeto de enseñanza, atravesando uno o más modelos intermediarios (Clement 2000). En función de ello, en esta investigación se diseñó una SEA dirigida a estudiantes de nivel primario cuya meta fue la construcción de un modelo topocéntrico con características descriptivas, explicativas y predictivas sobre el fenómeno de las estaciones.

\section{Explicación topocéntrica de las estaciones del año}

Dado que no suele encontrarse en la mayor parte de los materiales educativos, a continuación se desarrolla una síntesis de un modelo cinemático celeste topocéntrico explicativo del fenómeno de las estaciones del año para un observador situado en latitudes medias del hemisferio sur. Dado el carácter local de los movimientos celestes, se indican entre paréntesis los cambios que se deben realizar para que la explicación pueda utilizarse en latitudes medias del hemisferio norte.

Como etapa previa, es necesario desarrollar el ciclo día/noche explicándolo a partir del movimiento del Sol en el cielo. Desde el sistema de referencia topocéntrico, el día y la noche pueden comprenderse en forma sencilla como una consecuencia del movimiento diario que realiza el Sol desde el horizonte oriental al occidental: cuando se ubica por encima del horizonte local es de día, mientras que es de noche cuando se encuentra por debajo.

Por su parte, las estaciones del año pueden explicarse como una consecuencia del movimiento anual que realiza el Sol en el cielo de norte a sur y de sur a norte a lo largo del año. Si observamos su posición en el cielo cerca del día 21 de diciembre, notaremos que el Sol sale y se pone lo más al sur del este y del oeste, respectivamente, de todo el año. En consecuencia, en el hemisferio sur (norte) su altura en el mediodía solar será la más alta (baja) de todo el año y, por lo tanto, una estaca vertical proyectará su sombra anual más corta (larga). Esta fecha de solsticio de diciembre marca el comienzo del verano (invierno) en el hemisferio sur (norte).

A partir de ese día, el Sol comienza a moverse en el cielo cada día más hacia el norte hasta que, cerca del 21 de junio, el Sol realiza sus salidas y puestas lo más norte del este y del oeste, respectivamente, de todo el año. Por lo tanto, en el hemisferio sur (norte) el Sol recorre ese día un arco que es el más bajo (alto) de todo el año, por lo que una estaca vertical proyectará su sombra anual más larga (corta) en el mediodía solar. Este solsticio de junio marca el comienzo del invierno (verano) en el hemisferio sur (norte).

Desde el 21 de junio al 21 de diciembre, las salidas del Sol se van desplazando cada vez más hacia el sur, por lo que recorre arcos diurnos más altos (bajos). Durante ese lapso, existe un solo día en el que el Sol sale exactamente por el este y se pone por el oeste: el 22 o 23 de septiembre, día del equinoccio de septiembre y comienzo de la primavera (otoño) en el hemisferio sur (norte). En esa fecha, el Sol realiza la mitad de su recorrido por encima del horizonte y la otra mitad por debajo del mismo, razón por la cual el día tiene 12 horas de luz y 12 horas de oscuridad. De ahí proviene la palabra equinoccio, que significa «igual duración» o «igual noche». 
Durante el período que va desde el 21 de diciembre al 21 de junio, el Sol realiza el movimiento opuesto, de sur a norte, saliendo en consecuencia cada día más tarde (temprano) y recorriendo arcos cada vez más bajos (altos). Durante dicho período, existe un solo día en el que el Sol sale justo por el este y se oculta por el punto geográfico oeste: el 20 o 21 de marzo, día del equinoccio de marzo y comienzo del otoño (primavera) en el hemisferio sur (norte).

En la figura 1 se representan los cambios anuales en la trayectoria diaria del Sol para una localidad ubicada en una latitud media del hemisferio sur y sus consecuencias asociadas: modificación de la altura del Sol al mediodía y de los horarios y lugares de su salida y puesta. Se visualiza que en el hemisferio sur las trayectorias diarias se encuentran inclinadas hacia el norte. Por el contrario, se encuentran inclinadas hacia el sur en el hemisferio norte. Esta inclinación de las trayectorias respecto a la vertical posee el mismo valor que la latitud del lugar, siendo la variación de la altura solar una consecuencia de la esfericidad terrestre.

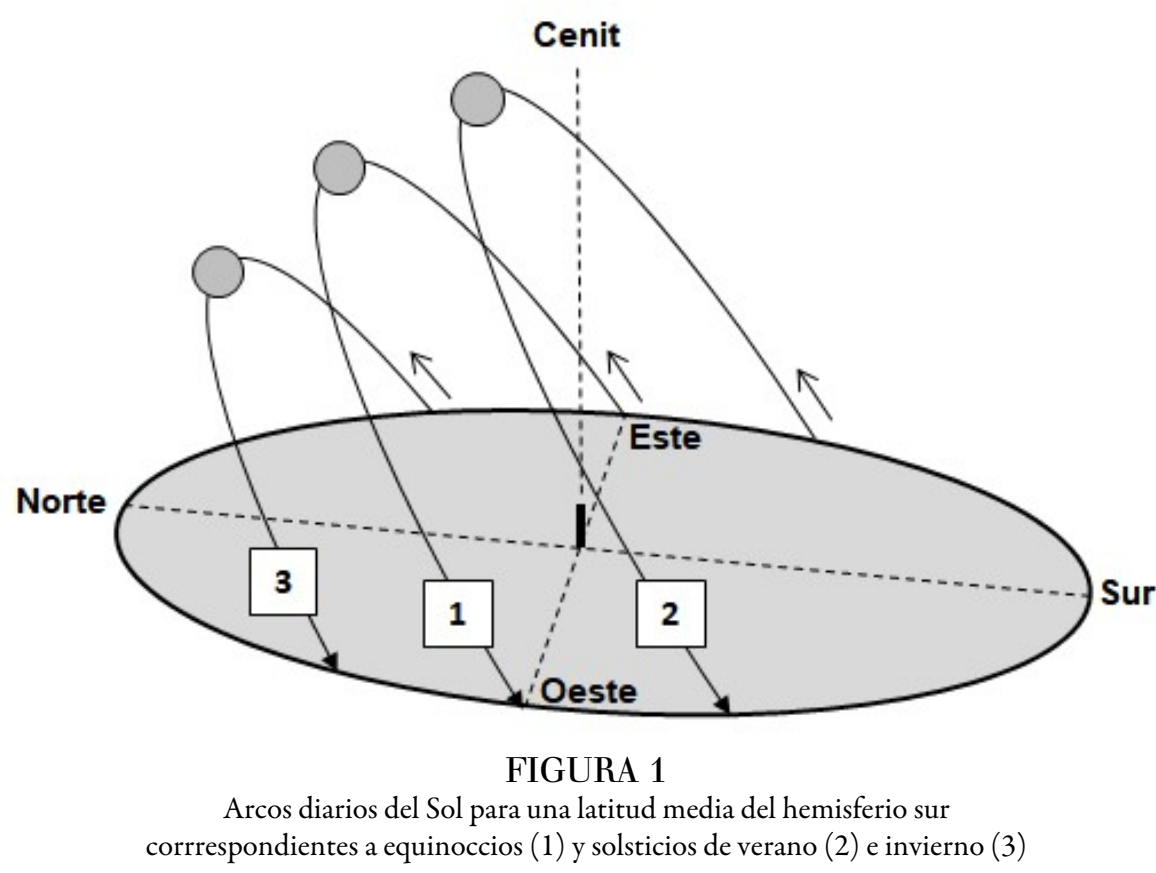

De este modo, el movimiento anual del Sol en el cielo permite explicar topocéntricamente las estaciones del año a partir de los cambios en la altura del Sol y en la cantidad de horas en que éste se ubica por encima del horizonte local: cuando el Sol se ubica más bajo en el cielo (en otoño e invierno) sus rayos llegan más inclinados y, en consecuencia, inciden sobre un área mayor, disminuyendo la radiación recibida por unidad de superficie. En cambio, cuando el Sol se ubica más alto (en primavera y verano), los rayos inciden menos inclinados respecto a la vertical y por lo tanto, se distribuyen en una superficie menor (figura 2). 


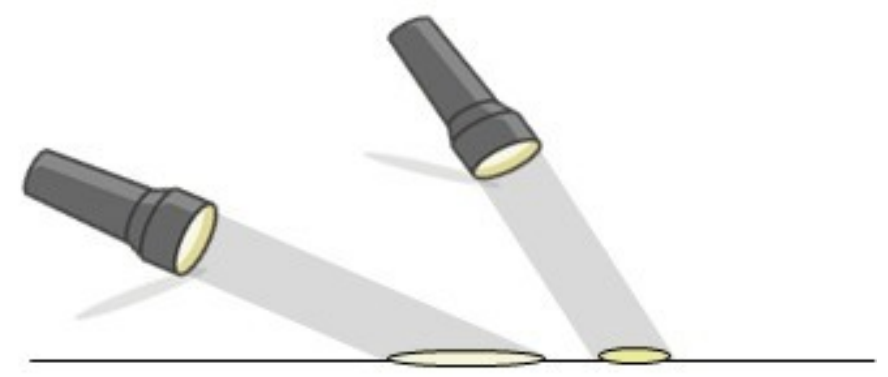

FIGURA 2

Analogía explicativa de los cambios en la radiación por unidad de superficie según la inclinación de los rayos. La energía solar se distribuye en una superficie mayor en invierno (izquierda) que en verano (derecha)

Las variaciones anuales en la trayectoria diaria que sigue el Sol en el cielo se deben al paulatino cambio de su posición en la esfera celeste. Este movimiento se produce sobre el plano de la eclíptica, el cual se encuentra inclinado $23,5^{\circ}$ respecto al Ecuador celeste. En consecuencia, el Sol se desplaza en el cielo $23,5^{\circ}$ al norte y al sur del Ecuador, ubicación que corresponde a los trópicos de Capricornio (hemisferio sur) y Cáncer (hemisferio norte). Esto provoca que los lugares de nuestro planeta que se encuentran entre ambos trópicos tengan el Sol justo sobre sus cabezas en dos momentos del año durante el mediodía solar. En la figura 3 se representa el movimiento anual del Sol entre los trópicos, lo que permite explicar por qué ocurren estaciones opuestas entre los hemisferios. A su vez, el esquema permite predecir aspectos observables del movimiento diario del Sol con solo colocar una estaca imaginaria en la posición en la que se desea conocer cómo se observará el desplazamiento solar desde allí.

Por ejemplo, si se desea conocer cómo se desplaza el Sol en el cielo en una ciudad como Buenos Aires, situada a unos $35^{\circ}$ de latitud sur, se debe marcar un punto en la figura 3 aproximadamente en dicha latitud (al sur del Trópico de Capricornio) sobre la dirección norte-sur indicada verticalmente por los soles. De ese modo, el punto marcado indicará la colocación de una estaca en Buenos Aires y los soles dibujados mostrarán dónde se ubica el Sol en el horario del mediodía solar durante los solsticios y equinoccios, sabiendo que en otras fechas estará en posiciones intermedias. El esquema muestra que los lugares de salida y puesta del Sol no dependen de la ubicación del observador, pero sí la posición del Sol en el mediodía solar: en Buenos Aires siempre se ubicará hacia el norte en ese instante, por lo que la sombra de una estaca vertical siempre apuntará hacia el sur. De este modo, este esquema complementa la figura 1 al permitir deducir cómo cambian esos recorridos al observar desde otras ubicaciones. 


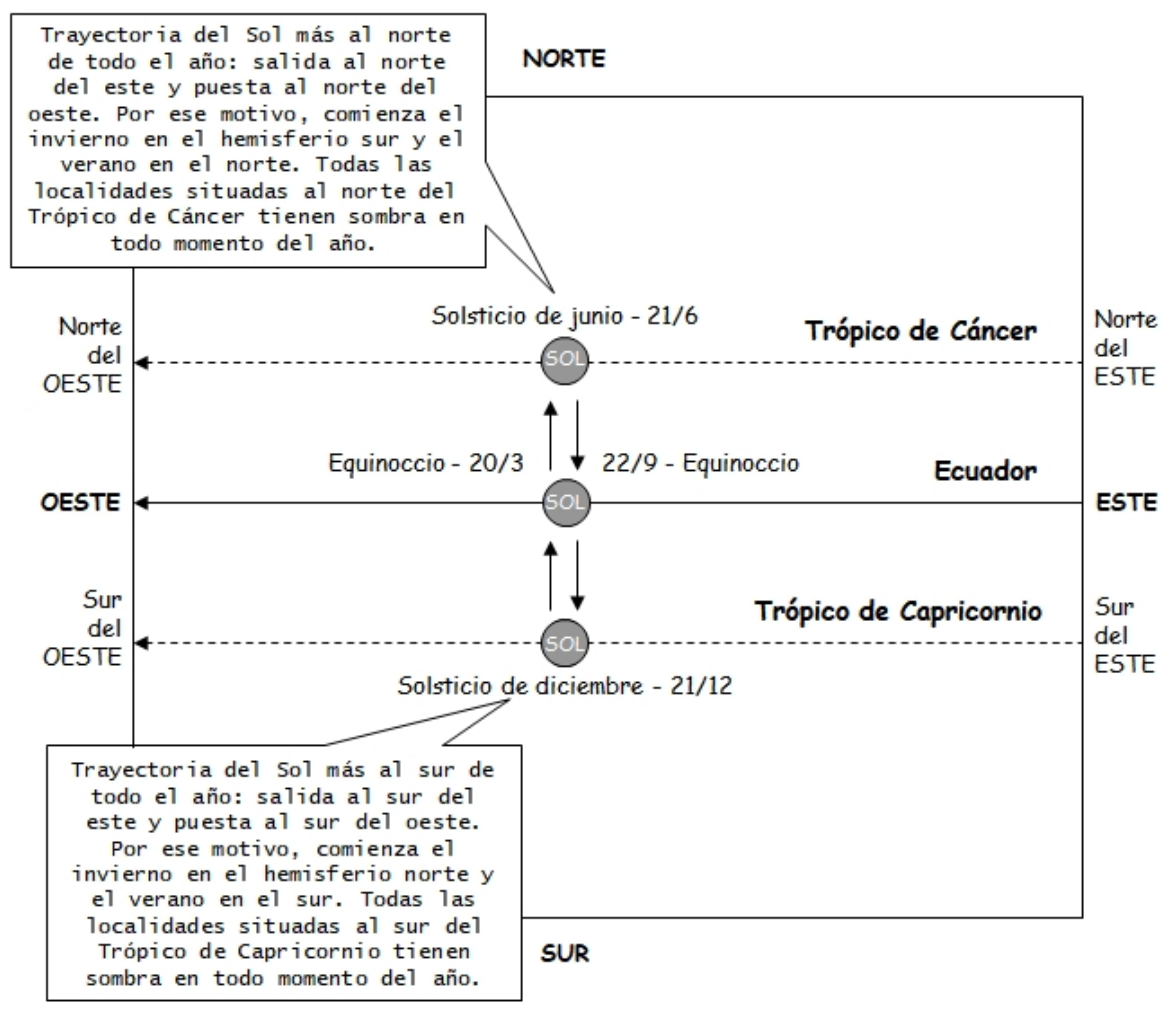

\section{FIGURA 3}

Representación simplificada del movimiento anual del Sol entre los trópicos. Permite explicar los cambios en los lugares de salida y puesta del Sol y, a su vez, en la longitud y dirección de la sombra de una estaca vertical ubicada en distintas latitudes en el instante del mediodía solar (Galperin 2011)

\section{Diseño de la secuencia de enseñanza-aprendizaje}

La SEA desarrollada tuvo como propósito la construcción de un modelo escolar topocéntrico que permita que los estudiantes comprendan el fenómeno de las estaciones del año a partir del movimiento anual del Sol en el cielo. Como etapa previa a esta propuesta, se llevaron a cabo actividades relativas a la observación y el registro del movimiento diario del Sol en el cielo para, a partir del mismo, construir una explicación topocéntrica del ciclo día/noche: en función de la presencia o no del Sol por encima del horizonte local. Para simplificar, durante dicha etapa se optó por describir el movimiento diario del Sol en las fechas de equinoccios, de este a oeste, dejando de lado los cambios anuales que ocurren en los recorridos diurnos.

En función del análisis bibliográfico previo, la SEA comienza con actividades con el fin de conocer, explicitar y analizar las concepciones presentes en los estudiantes en relación a la causa de las estaciones del año. A partir de allí se realizan descripciones y registros de las variaciones que ocurren en el movimiento diario del Sol en el cielo, empleando simulaciones que se llevan a cabo con el programa Stellarium (www.stellarium.org), en forma simultánea con observaciones realizadas desde la escuela y desde la propia casa de los estudiantes.

Estas actividades tienen como propósito visualizar los cambios en los lugares y horarios de salida y puesta del Sol a lo largo del año y relacionarlos con las modificaciones en la altura del Sol en el mediodía solar. Posteriormente, se utilizan los datos obtenidos con el fin de construir los recorridos diarios correspondientes a solsticios y equinoccios, se presentan esquemas explicativos del fenómeno y se resuelven actividades grupales. En esta última etapa, los estudiantes construyen una maqueta para representar dichos recorridos con el fin de explicar el fenómeno en un modelo concreto de carácter local. Finalmente, ponen en juego sus 
nuevas ideas al realizar la actividad inicial nuevamente y al resolver consignas de evaluación. En la tabla 2 se presenta una síntesis del diseño de la SEA incluyendo las consignas generales de cada actividad y las preguntas guía de cada una. La secuencia completa puede consultarse aquí (https://1drv.ms/b/s!AuvrlNHB0HiRt3L ar9tORSSYtinP?e=ETQyZj).

TABLA 2

Síntesis de la SEA y de su contenido didáctico

\begin{tabular}{|c|c|c|c|c|}
\hline Nro & Nombre & Consigna/s & Pregunta/s & Síntesis \\
\hline $\begin{array}{c}\text { Act. } \\
1\end{array}$ & $\begin{array}{l}\text { Poniendo en } \\
\text { juego nuestras } \\
\text { ideas sobre las } \\
\text { estaciones }\end{array}$ & $\begin{array}{l}\text { Explicar las estaciones del } \\
\text { año mediante un dibujo y un } \\
\text { texto. Explicitación oral }\end{array}$ & $\begin{array}{c}\text { ¿A qué te parece que se } \\
\text { deben las estaciones del } \\
\text { año? }\end{array}$ & $\begin{array}{l}\text { Indagación de las ideas de } \\
\text { los estudiantes sobre las } \\
\text { estaciones del año y puesta } \\
\text { en común de las mismas }\end{array}$ \\
\hline $\begin{array}{c}\text { Act. } \\
2\end{array}$ & $\begin{array}{l}\text { Movimiento } \\
\text { anual del Sol }\end{array}$ & $\begin{array}{l}\text { Completar una tabla con } \\
\text { datos sobre los lugares y } \\
\text { horarios de salida del Sol } \\
\text { usando Stellarium. Observa- } \\
\text { ción de estos cambios desde } \\
\text { la escuela o desde las casas }\end{array}$ & $\begin{array}{l}\text { ¿Cómo se desplazan las } \\
\text { salidas y las puestas del } \\
\text { Sol durante el año? } \\
\text { ¿Cambian los horarios? } \\
\text { ¿ué relación tiene con } \\
\text { las estaciones del año? }\end{array}$ & $\begin{array}{l}\text { Descripción del movimien- } \\
\text { to anual del Sol usando } \\
\text { Stellarium y observación } \\
\text { directa del cielo. } \\
\text { Corrección de actividad } \\
\text { final de comprensión }\end{array}$ \\
\hline $\begin{array}{c}\text { Act. } \\
3\end{array}$ & $\begin{array}{l}\text { Comprendien- } \\
\text { do los cam- } \\
\text { bios anuales } \\
\text { en el recorrido } \\
\text { del Sol }\end{array}$ & $\begin{array}{l}\text { Utilizar el esquema de los } \\
\text { cambios en el recorrido } \\
\text { diario del Sol para completar } \\
\text { un cuadro sobre lugares, } \\
\text { horarios y alturas del Sol }\end{array}$ & $\begin{array}{l}\text { ¿Qué relación hay entre } \\
\text { el lugar de salida y } \\
\text { puesta del Sol, la } \\
\text { cantidad de horas de } \\
\text { luz y la altura del Sol? }\end{array}$ & $\begin{array}{l}\text { Explicación del } \\
\text { movimiento anual del Sol y } \\
\text { presentación de esquema } \\
\text { descriptivo. Corrección de } \\
\text { actividad de comprensión }\end{array}$ \\
\hline $\begin{array}{c}\text { Act. } \\
4\end{array}$ & $\begin{array}{l}\text { Construyendo } \\
\text { la explicación } \\
\text { topocéntrica } \\
\text { de las } \\
\text { estaciones }\end{array}$ & $\begin{array}{c}\text { Utilizar la explicación } \\
\text { topocéntrica de las estaciones } \\
\text { del año para resolver en } \\
\text { forma grupal una actividad } \\
\text { de completamiento }\end{array}$ & $\begin{array}{l}\text { ¿Cómo se explican las } \\
\text { estaciones del año a } \\
\text { partir del movimiento } \\
\text { anual del Sol? }\end{array}$ & $\begin{array}{l}\text { Explicación de las } \\
\text { estaciones del año a partir } \\
\text { del movimiento anual del } \\
\text { Sol. Corrección de } \\
\text { actividad grupal de } \\
\text { comprensión }\end{array}$ \\
\hline $\begin{array}{c}\text { Act. } \\
5\end{array}$ & $\begin{array}{l}\text { Construcción } \\
\text { de un modelo } \\
\text { concreto para } \\
\text { explicar las } \\
\text { estaciones }\end{array}$ & $\begin{array}{l}\text { Construcción de una } \\
\text { maqueta que represente el } \\
\text { movimiento anual del Sol. } \\
\text { Utilizarla para explicar } \\
\text { topocéntricamente las } \\
\text { estaciones del año }\end{array}$ & $\begin{array}{l}\text { ¿Cómo se puede } \\
\text { utilizar el desplaza- } \\
\text { miento anual del Sol } \\
\text { para explicar las } \\
\text { estaciones del año? }\end{array}$ & $\begin{array}{l}\text { Realización de un modelo } \\
\text { concreto explicativo de las } \\
\text { estaciones a partir del } \\
\text { movimiento anual del Sol. } \\
\text { Presentación grupal }\end{array}$ \\
\hline $\begin{array}{c}\text { Act. } \\
6\end{array}$ & $\begin{array}{l}\text { Evaluando los } \\
\text { conocimientos } \\
\text { adquiridos en } \\
\text { forma grupal }\end{array}$ & $\begin{array}{l}\text { Realización grupal de una } \\
\text { explicación de las estaciones } \\
\text { del año mediante un dibujo } \\
\text { complementado con un texto }\end{array}$ & $\begin{array}{l}\text { ¿Por qué se producen } \\
\text { las estaciones del año? } \\
\text { ¿Es posible explicarlo } \\
\text { en forma topocéntrica? }\end{array}$ & $\begin{array}{l}\text { Presentación grupal de } \\
\text { dibujos explicativos de las } \\
\quad \text { estaciones del año. } \\
\text { Evaluación de las mismos }\end{array}$ \\
\hline $\begin{array}{c}\text { Act. } \\
7\end{array}$ & $\begin{array}{l}\text { Evaluando los } \\
\text { conocimientos } \\
\text { adquiridos en } \\
\text { forma } \\
\text { individual }\end{array}$ & $\begin{array}{l}\text { Resolución individual de } \\
\text { consignas de evaluación de } \\
\text { conocimientos. Autorevisión } \\
\text { de la explicación inicial dada } \\
\text { en forma de dibujo y texto }\end{array}$ & $\begin{array}{l}\text { ¿Cómo cambia el } \\
\text { recorrido diario del Sol } \\
\text { en el cielo? ¿Cuál es su } \\
\text { relación con las } \\
\text { estaciones del año? }\end{array}$ & $\begin{array}{l}\text { Resolución de consignas de } \\
\text { comprensión de la causa de } \\
\text { las estaciones a partir del } \\
\text { movimiento anual del Sol. } \\
\text { Corrección de las mismas }\end{array}$ \\
\hline
\end{tabular}




\section{Metodología}

Como primera etapa de la IBD, se diseñó la SEA y se llevó a cabo un proceso inicial de implementación con el fin de extraer conclusiones y modificaciones para futuras iteraciones. Para ello se utilizó un enfoque de investigación-acción en el que el investigador actuó como docente del curso y como investigador de su propia práctica y del grado de incidencia de la misma en la realidad escolar (Sandín Esteban 2003). Al mismo tiempo, el docente del curso actuó como observador de las actividades, lo que permitió revisar constantemente la secuencia y realizar el análisis posterior del proceso de implementación.

El estudio llevado a cabo se incluye dentro de un marco de investigación cualitativa debido a que el mismo no pretende corroborar teorías ni extender generalizaciones; por el contrario, se pone el acento en la búsqueda de interpretaciones y asignación de significados dentro de un contexto particular (Bryman 2004). En este sentido, se buscó conocer el impacto que posee una SEA diseñada desde un punto de vista topocéntrico en la comprensión del fenómeno de las estaciones del año por parte de un grupo concreto de estudiantes de nivel primario.

En esta primera etapa participó un curso de sexto año de nivel primario perteneciente a una escuela pública de educación primaria situada en la zona urbana de la localidad de El Bolsón, Argentina. El curso estaba integrado por 22 alumnos de entre 9 y 11 años $(M=10.6, D E=0.6)$. La mayoría de los estudiantes pueden caracterizarse como de clase media.

Después de implementar la SEA y siguiendo el enfoque IBD, la misma fue evaluada a partir del análisis de los trabajos realizados durante el proceso de implementación, de la realización de entrevistas semiestructuradas y de la clasificación y el análisis de la evolución de los modelos mentales de los estudiantes sobre la causa de las estaciones del año. Con el fin de llevar a cabo dicho análisis se registraron las clases en formato de audio y video, se tomaron fotografías y se recogieron la totalidad de los trabajos llevados a cabo por los estudiantes, tanto en forma individual como grupal. Esto permitió conocer el proceso de aprendizaje de cada estudiante, para lo cual se organizaron y evaluaron las transcripciones de todas las conversaciones de aula. También se llevaron cabo entrevistas semiestructuradas con ocho de los estudiantes, tanto al inicio como al finalizar la implementación. Esto permitió caracterizar el proceso de evolución de los modelos mentales de los estudiantes y, de ese modo, establecer la efectividad de la propuesta.

\section{Resultados}

A continuación se presentan en forma sintética los resultados obtenidos. Para ello y con el fin de evaluar el diseño de la SEA, se centró la atención en si la misma y su posterior proceso de implementación propiciaron la evolución de los modelos mentales de los estudiantes en relación a una mejora en la comprensión del fenómeno de las estaciones del año y en la motivación del alumnado por conocer y comprender los fenómenos celestes posibles de ser percibidos desde sus propias casas.

\section{Concepciones iniciales de los estudiantes sobre la causa de las estaciones}

A partir del análisis de la actividad 1 realizada por todo el curso $(\mathrm{N}=22)$, se visualizó que el 45,5\% de los estudiantes dibujaron cambios en el ambiente (lluvias, nieve, calor, etc) como forma de brindar una explicación de las estaciones del año. A su vez, 36,3\% de los estudiantes brindaron explicaciones en las que asociaban el fenómeno con la distancia variable entre la Tierra y el Sol. De los restantes alumnos, un 13,6\% brindó respuestas confusas de difícil clasificación, mientras que un 4,6\% no dio ninguna respuesta. Mediante el análisis de las respuestas iniciales se definieron dos categorías de modelos mentales explicativas de las estaciones del año: a) por cambios ambientales $\left(\mathrm{M}_{\mathrm{EA}-1}\right)$ y b) a partir de la variación de la distancia Tierra- 
Sol ( $\left.\mathrm{M}_{\mathrm{EA}-2}\right)$. A su vez, dentro de la categoría $\mathrm{M}_{\mathrm{EA}-2}$ se definieron 3 subcategorías explicativas de por qué se modifica la distancia al Sol. La tabla 3 brinda ejemplos de respuestas de los estudiantes y su presencia porcentual al realizar la actividad 1 de la SEA.

TABLA 3

Categorías de modelos mentales sobre las estaciones del año definidas a partir de las explicaciones orales, escritas y pictóricas de los estudiantes al inicio de la secuencia. Se brindan ejemplos de explicaciones

\begin{tabular}{|c|c|}
\hline 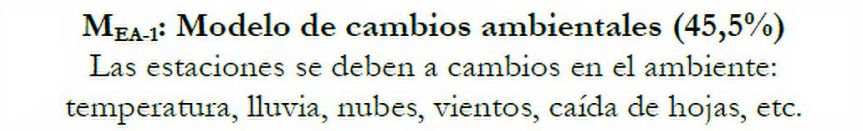 & $\begin{array}{c}\text { «Porque en invierno hace frío y llueve mucho. } \\
\text { En otoño se caen las hojas y en verano hace } \\
\text { mucho calon }\left(\mathrm{A}_{3}\right)\end{array}$ \\
\hline 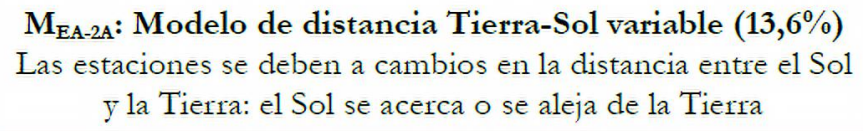 & «Es verano porque el Sol está más cerca» $\left(A_{13}\right)$ \\
\hline $\begin{array}{l}\text { M }_{\text {EA-2B }} \text { : Modelo de distancia Tierra-Sol variable }(\mathbf{9}, 1 \%) \\
\text { Las estaciones se deben a cambios en la distancia Sol-Tierra: } \\
\text { al moverse en su órbita, la Tierra se acerca o se aleja del Sol }\end{array}$ & $\begin{array}{l}\text { «La Tierra gira alrededor del Sol como un } \\
\text { óvalo. Cuando está más cerca hace más calor, } \\
\text { y cuando está más lejos más frío» }\left(\mathrm{A}_{23}\right)\end{array}$ \\
\hline $\begin{array}{l}\text { M }_{\text {EA-2c: }} \text { Modelo de distancia Tierra-Sol variable }(13,6 \%) \\
\text { E1 Sol se acerca a la Tierra cuando la Luna se aleja y viceversa }\end{array}$ & $\begin{array}{l}\text { «Invierno: Sol muy lejos y Luna muy cerca. } \\
\text { Verano: Sol cerca y Luna más lejos» }\left(A_{19}\right)\end{array}$ \\
\hline
\end{tabular}

En conclusión, la mayoría de las respuestas de los estudiantes pueden categorizarse dentro de dos modelos explicativos inadecuados: el que asocia las estaciones del año con variaciones ambientales, confundiendo la causa del fenómeno con sus consecuencias, y el que explica las estaciones en función de cambios en la distancia al Sol. Estos últimos modelos de distancia Tierra-Sol variable guardan relación con situaciones cotidianas en las cuales los objetos son acercados a una fuente de calor para su calentamiento. Las distintas subcategorías dentro de este modelo se relacionan con el modo en que los estudiantes explican el acercamiento o alejamiento Tierra-Sol: a) por un movimiento del Sol, b) por la traslación de la Tierra en su órbita «ovalada» o c) por un desplazamiento del Sol opuesto a la Luna. Por lo tanto, puede concluirse que, al inicio de la implementación de la SEA, la totalidad de los estudiantes del curso brinda explicaciones alejadas del conocimiento científico sobre la causa de las estaciones.

\section{Evolución de los modelos mentales de los estudiantes}

Como etapa final de la SEA, los estudiantes desarrollaron actividades para utilizar sus nuevos conocimientos sobre el fenómeno de las estaciones del año, lo que permitió analizar la evolución de sus modelos mentales. En este sentido, en la actividad 6 se elaboraron dibujos grupales explicativos en los cuales, a diferencia de lo sucedido en la actividad 1, la totalidad de los grupos explicitaron la relación entre las estaciones del año y el cambio de lugar de salida y puesta del Sol: al norte en invierno y al sur en verano (figura 4). Estas representaciones indican un salto conceptual significativo en relación a lo sucedido al inicio de la SEA, donde ninguno de los estudiantes pudo brindar una explicación adecuada acerca del fenómeno. 


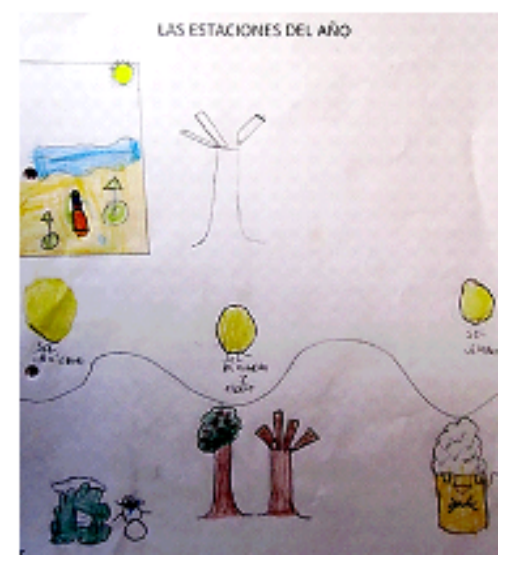

FIGURA 4

Explicación grupal final. Se visualiza la relación entre el lugar de salida del Sol y la estación del año

Posteriormente, con el fin de analizar la evolución conceptual del grupo de clase durante la implementación de la SEA, cada estudiante resolvió individualmente la actividad 7. En la misma se debían resolver consignas de comprensión y, a su vez, llevar a cabo nuevamente la actividad inicial de explicación del fenómeno mediante un dibujo acompañado de un texto. Posteriormente, tenían que compararlo con el que habían realizado al inicio. En esta instancia, todos los estudiantes elaboraron dibujos y textos en las que asociaron las estaciones con el cambio anual en la posición del Sol, por lo que sus respuestas fueron clasificadas dentro de una nueva categoría de modelo mental: Modelo de variación anual de la posición del Sol ( $\left.\mathrm{M}_{\mathrm{EA}-3}\right)$. Esto permitió detectar una evolución conceptual favorable en la comprensión del fenómeno al haberse observado un cambio radical en el modelo utilizado. En la tabla 4 se presentan ejemplos representativos de dicha evolución conceptual: las representaciones iniciales son diversas e inadecuadas, mientras que las finales asocian las estaciones con los cambios en el lugar de salida del Sol. 
TABLA 4

Evolución conceptual. Dibujos al inicio y al final de la SEA y frases comparativas de los estudiantes

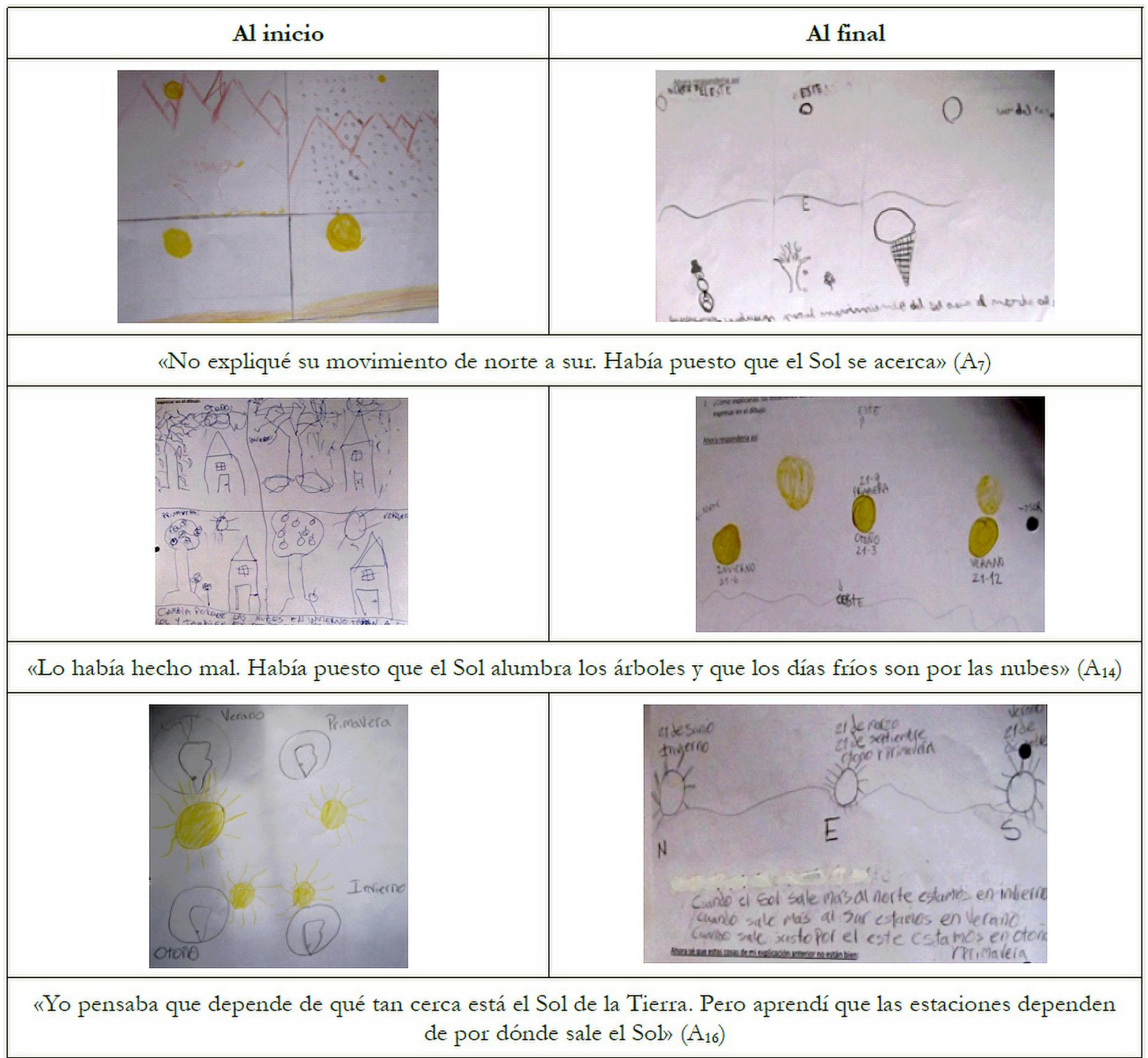

Esta evolución de las representaciones internas acerca del fenómeno quedó en evidencia en las entrevistas individuales finales, donde los estudiantes pudieron explicar las estaciones del año a partir del movimiento anual del Sol en el cielo e indicar cuestiones descriptivas y explicativas:

«En verano el Sol sale más temprano y llega a su punto más alto en el cielo; o sea, no está arriba de nuestras cabezas, está a un costado.» (A.)

«El Sol va saliendo más al norte del este o más al sur. Cuando sale más al norte, acá que vivimos en el sur esos días es invierno porque los rayos llegan más inclinados. Y cuando sale más al sur es verano porque llegan más verticales.» $\left(\mathrm{A}_{13}\right)$

«Dibujé un Sol de verano más hacia el sur, un Sol de primavera y otoño justo en el este y uno de invierno hacia el norte. Como nosotros estamos al sur de la Tierra y los rayos del Sol nos pegan inclinados, hace que sintamos menos calor.» $\left(\mathrm{A}_{19}\right)$

«Verano es cuando el Sol está más al sur y nos dan más los rayos. Cuando está al norte es invierno. Cuando está en el medio es otoño o primavera. En invierno los rayos llegan muy inclinados y las horas de luz son pocas.» $\left(\mathrm{A}_{22}\right)$ 
De acuerdo con estos resultados y del análisis de las respuestas dadas en las consignas de comprensión, es posible concluir que la SEA resultó efectiva para lograr una evolución de los modelos explicativos de los estudiantes en relación al fenómeno de las estaciones del año. A su vez, en las entrevistas y durante las mismas clases, los estudiantes expresaron su interés en la temática y en la observación del cielo, lo que quedó plasmado en distintos registros gráficos y orales de observaciones llevadas a cabo relativas a los lugares y horarios de salida y puesta del Sol. Sin embargo, la corrección de la actividad 7 mostró cierta disparidad de los estudiantes en la posibilidad de explicar la relación entre las distintas variables involucradas: lugar y horario de salida y puesta del Sol, recorrido diario, altura al mediodía, longitud de la sombra de una estaca al mediodía, etc. Por lo tanto, el análisis llevado a cabo permitió proponer modificaciones a realizar en la SEA para ser evaluadas en el siguiente proceso de implementación.

\section{CONCLUSIONES Y LÍNEAS FUTURAS}

El objetivo de este trabajo fue describir la etapa inicial de una IBD a partir de la evaluación de una SEA sobre el fenómeno de las estaciones del año. La misma fue diseñada utilizando el sistema de referencia topocéntrico, lo que implica plantear la explicación del fenómeno como una consecuencia del movimiento anual del Sol en el cielo. Los resultados hallados sugieren que este enfoque promueve una evolución favorable de los modelos mentales de los estudiantes y, a su vez, permite vincular los conocimientos astronómicos de los alumnos con los fenómenos que pueden percibir a diario en el cielo. Sin embargo, algunos estudiantes tuvieron dificultades para poder relacionar las distintas variables involucradas (posición del Sol, horarios, longitud de la sombra, fechas, etc), por lo que se propusieron dos modificaciones para continuar el proceso iterativo de nuevas implementaciones y evaluaciones: a) incluir una mayor realización de observaciones del cielo y de explicaciones utilizando el propio entorno natural del patio escolar y b) un trabajo más extenso con el modelo concreto realizado por los estudiantes, utilizándolo para explicar y predecir diferentes situaciones astronómicas cotidianas. Dichas modificaciones han sido introducidas en una nueva versión de la SEA que será implementada y evaluada en otro curso de nivel primario.

El desarrollo topocéntrico presentado puede ser utilizado como modelo intermediario en la construcción de un modelo objetivo que ubique al observador fuera de la Tierra para explicar el mismo fenómeno heliocéntricamente. Sin embargo, más allá de constituir una posible transición hacia un modelo más complejo, la SEA desarrollada ha mostrado poseer su propio valor educativo al haber contribuido a la evolución de los modelos mentales de los estudiantes, permitiendo la descripción, explicación y predicción de un fenómeno astronómico poco comprendido pese a su cotidianeidad.

El enfoque cualitativo centrado en el desarrollo de secuencias de enseñanza contextualizadas utilizado en esta IBD no pretende generalizar sus resultados ni que sean transferibles del mismo modo a otras situaciones de enseñanza. Sin embargo, la descripción detallada de la SEA y de su proceso de implementación permite su utilización como hipótesis de trabajo por parte de otros investigadores y docentes con el fin de generar otras propuestas que permitan vincular la enseñanza de la astronomía con lo que puede observarse a simple vista en el cielo.

\section{ReFERENCIAS}

Alvarez M., Galperin D., Quinteros C. (2018) Indagación de las concepciones de estudiantes primarios y secundarios sobre los fenómenos astronómicos cotidianos. En Papini, M. (comp.), Las ciencias de la naturaleza y la matemática en el aula: nuevos desafíos y paradigmas, 129-142. Tandil: UNICEN.

Baxter J. (1989) Children's understanding of familiar astronomical events. International Journal of Science Education 11(5), 502-513. 
Black A. (2005) Spatial Ability and Earth Science Conceptual Understanding. Journal of Geoscience Education 53(4), 402-414.

Bryman A. (2004) Social research methods. New York: Oxford University Press.

Camino N. (1995) Ideas previas y cambio conceptual en astronomía. Un estudio con maestros de primaria sobre el día y la noche, las estaciones y las fases de la Luna. Enseñanza de las Ciencias 13(1), 81-96.

Camino N. (1999) Sobre la didáctica de la astronomía y su inserción en EGB. En Kaufman M., Fumagalli L. (comps.) Enseñar Ciencias Naturales, 143-173. Buenos Aires: Paidós.

Clement J. (2000) Model based learning as a key research area for science education. International Journal of Science Education 9 (22), 1041-1053.

Danaia L., McKinnon, D. (2007) Common alternative astronomical conceptions encountered in junior secondary science classes: Why is this so? Astronomy Education Review 6(2), 32-53.

De Manuel J. (1995) ¿Por qué hay veranos e inviernos? Representaciones de estudiantes y de futuros maestros sobre algunos aspectos del modelo Sol-Tierra. Enseñanza de las Ciencias 13(2), 227-236.

Fernández Nistal M., Peña Boone S. (2007) Concepciones de maestros de primaria sobre el día y la noche y las estaciones del año. Revista Latinoamericana de Estudios Educativos 37(3-4), 189-220.

Galagovsky L., Adúriz-Bravo A. (2001) Modelos y analogías en la enseñanza de las ciencias naturales. El concepto de modelo didáctico analógico. Enseñanza de las ciencias 19(2), 231-242.

Gali F., Venukapalli S. (2021) Does visual-spatial cognition affect children's astronomical experiences? European Journal of Education Studies 8(1), 179-195.

Galperin D. (2011). Propuestas didácticas para la enseñanza de la Astronomía. En Insaurralde, M. (coord.) Ciencias Naturales. Lineas de acción didáctica y perspectivas epistemológicas, 189-229. Buenos Aires: Novedades Educativas.

Galperin D., Raviolo A. (2014) Sistemas de referencia en la enseñanza de la Astronomía. Un análisis a partir de una revisión bibliográfica. Latin American Journal of Physics Education 8(1), 136-148.

Galperin D., Raviolo A. (2015) Argentinean students' and teachers' conceptions of day and night: an analysis in relation to astronomical reference systems. Science Education International 26(2), 126-147.

Galperin D., Raviolo A. (2017) Análisis de imágenes relacionadas con día/noche, estaciones y fases lunares en textos de enseñanza primaria. Revista Electrónica de Investigación en Educación en Ciencias 12(1), 1-11.

Galperin D., Prieto L., Heredia L. (2018) Concepciones de docentes sobre las causas de los fenómenos astronómicos cotidianos. En Papini, M. (comp.), Las ciencias de la naturaleza y la matemática en el aula: nuevos desafíos y paradigmas, 116-128. Tandil: UNICEN.

Galperin D., Alvarez M., Heredia L., Haramina J. (2020) Análisis de videos educativos y de divulgación sobre día/ noche, estaciones y fases lunares. Revista Enseñanza de la Física 32(no. extra), 125-133.

García Barros S., Martínez Losada C., Mondelo M. (1996) La astronomía en la formación de profesores. Alambique. Didáctica de las Ciencias Experimentales 10, 121-127.

García Barros S., Mondelo M., Martínez Losada C., Larrosa Cañestro I. (2003) La observación del cielo. Un instrumento para estudiar el espacio y el tiempo. Suplemento Aula 51 40, 2-18. Barcelona: Graó Educación.

Gilbert J., Boulter C. (eds.) (2000) Developing models in science education. Dordrecht: Kluwer.

Guisasola J., Amettler J., Zuza K. (2020) Investigación basada en el diseño de Secuencias de Enseñanza-Aprendizaje: una línea de investigación emergente en Enseñanza de las Ciencias. Revista Eureka sobre Enseñanza y Divulgación de las Ciencias 18(1), 1801.

Johnson-Laird P. (1983) Mental models. Cambridge: Cambridge University Press.

Kikas E. (1997) The impact of teaching on students's explanations of astronomical phemomena. Psychology of Language and Comunication 1 (2), 45-52.

Lanciano N. (1989) Ver y hablar como Tolomeo y pensar como Copérnico. Enseñanza de las Ciencias 7(2), 173-182.

Langhi R., Nardi R. (2007) Ensino de astronomia: erros conceituais mais comuns presentes em livros didáticos de ciências. Caderno Brasileiro de Ensino de Física 24(1), 87-111. 
Lombardi O. (1998) La noción de modelo en ciencias. Educación en Ciencias 2(4), 5-13.

Navarrete A., Azcárate P., Oliva J. M. (2004) Algunas interpretaciones sobre el fenómeno de las estaciones en niños, estudiantes y adultos: revisión de la literatura. Revista Eureka sobre Enseñanza y Divulgación de las Ciencias, 1(3), 146-166.

Navarro Pastor M. (2011) Enseñanza y aprendizaje de Astronomía diurna en primaria mediante "secuencias problematizadas" basadas en "mapas evolutivos". Enseñanza de las Ciencias 29(2), 163-174.

Nussbaum J. (1979) Childrens conceptions of the Earth as a cosmic body: a cross-age study. Science Education 63(1), 83-93.

Ojala J. (1992) The third planet. International Journal of Science Education 14(2), 191-200.

Parker J., Heywood D. (1998) The earth and beyond: developing primary teachers' understanding of basic astronomical events. International Journal of Science Education 20(5), 503-520.

Plomp T. (2010) Educational Design Research: An Introduction. En Plomp T., Nieveen N. (Ed) An Introduction to Educational Design Research. Proceedings of the seminar conducted at the East China Normal University, Shanghai (PR China).

Plummer J., Kocareli A., Slagle C. (2014) Learning to explain astronomy across moving frames of reference: Exploring the role of classroom and planetarium-based instructional contexts. International Journal of Science Education 36(7), 1083-1106.

Sandín Esteban M. (2003) Investigación cualitativa en educación: Fundamentos y tradiciones. Madrid: McGraw Hill.

Schoon K. (1992) Students alternative conceptions of Earth and space. Journal of Geological Education 40, 209-214.

Sharp J. (1996) Children's astronomical beliefs: a preliminary study of year 6 children in south-west England. International Journal of Science Education 18(6), 685-712.

Shen J., Confrey J. (2010) Justifying Alternative Models in Learning Astronomy: A study of K-8 science teacher's understanding of frames of reference. International Journal of Science Education 32 (1), 1-29.

Sneider C., Bar B., Kavanagh C. (2011) Learning about Seasons: A Guide for Teachers and Curriculum Developers. Astronomy Education Review 10(3), 1-22.

Trumper R. (2001) Assessing students' basic astronomy conceptions from junior high school through university. Australian Science Teachers Journal 47(1), 21-31.

Vega Navarro A. (1996) Ideas Precopernicanas en nuestros libros de texto. Revista de Educación 311, 339-354.

Vílchez-González J., Ramos-Tamajón C. (2015) La enseñanza-aprendizaje de fenómenos astronómicos cotidianos en la Educación Primaria española. Revista Eureka sobre Enseñanza y Divulgación de las Ciencias, 12(1), 2-21.

\section{INFORMACIÓN ADICIONAL}

Para citar este artículo: Galperin D. (2021) Diseño de una secuencia topocéntrica de enseñanza-aprendizaje de las estaciones del año para nivel primario. Revista Eureka sobre Enseñanza y Divulgación de las Ciencias 19(1), 1801.doi: 10.25267/Rev_Eureka_ensen_divulg_cienc.2022.v19.i1.1801 